\title{
Asymmetries in processing horizontal and vertical dimensions
}

\author{
ELISABETTA LÀDAVAS \\ University of Bologna, Bologna, Italy
}

\begin{abstract}
The aim of this study was to determine whether the referential term on the horizontal dimension corresponds to the dominant hand and whether the referential term on the vertical dimension is independent of handedness. In order to verify the hypothesis, right-handers, left-handers, and ambidextrous subjects were required to verify and falsify statements including the words ABOVE and BELOW and the words LEFT and RIGHT. The results showed that right-handers were faster in verifying and falsifying the statements containing the term RIGHT, whereas lefthanders were faster in verifying and falsifying those containing the term LEFT. Ambidextrous subjects, however, showed no sign of asymmetry in the positional judgments of stimuli along the horizontal dimension. By contrast, right-handers, left-handers, and ambidextrous subjects were equally faster in verifying and falsifying the statements containing the term $A B O V E$ than the statements containing the term BELOW. The relation between the positive term on the horizontal dimension and the dominant hand can be explained by the fact that, in the absence of any asymmetries in the physical world, the dominant hand can furnish a natural reference direction for judgments related to this dimension.
\end{abstract}

Numerous studies have shown that speed and accuracy of positional judgments of stimuli along the horizontal and vertical dimensions depend upon the referential axis. The difference occurs both across dimensions, in that discriminating locations on the horizontal axis appears more difficult than discriminating locations on the vertical axis (Corballis \& Beale, 1976; Farrell, 1979; Maki, Grandy, \& Hauge, 1979; Sholl \& Egeth, 1981), and within each dimension, since "above" positions appear to be processed faster than "below" positions (Chase \& Clark, 1971; Farrell, 1979; Seymour, 1969, 1974) and, although to a lesser extent, "right" positions appear to be processed faster than "left" positions (Farrell, 1979; Olson \& Laxar, 1973). The present paper addresses mainly the difference between the positional judgments of stimuli within each dimension, although one must keep in mind that the two aspects are not independent, but are both related to a general model of how human beings conceptualize space.

Clark (1973) suggested that asymmetry is an important factor in determining the manner in which the referential axis of a dimension is polarized. In other words, asymmetry seems to be responsible for the manner in which we distinguish between left and right and between above and below. But if the inherent asymmetry in the vertical dimension can explain why this dimension tends to be effectively polarized, it cannot account for the effect also

This research was supported by a leave grant from CNR-NATO to E. Ladavas and by Natural Sciences and Engineering Research Council of Canada Grant A8347 to Morris Moscovitch. I thank Morris Moscovitch for his precious help on the discussion of the research project and his kind friendship during my sabbatical year in his laboratory. Address correspondence to E. Làdavas, Department of Psychology, University of Bologna, Viale Berti Pichat 5, Bologna, Italy. found in the horizontal dimension. Whereas up and down can generally be specified in terms of reasonably constant physical characteristics, it is difficult to specify left and right in physical terms, because on the horizontal axis the everyday physical world seems generally symmetrical. Also the body is approximately symmetrical about the midsagittal plane and, therefore, does not have any salient physical characteristics upon which to base the definitions of left and right.

Even though the human body is nearly symmetrical about the midsagittal plane, most people have a dominant or preferred hand (right for right-handers and left for lefthanders) that could furnish a natural reference direction in the sagittal plane, just as height serves as a reference direction in the vertical plane. Therefore, it is possible to hypothesize that, in the absence of any asymmetry in the physical world, the referential term on the horizontal dimension may correspond to the dominant hand. On the contrary, the referential term on the vertical dimension cannot be related to handedness, because the two hands are asymmetrical along the horizontal and not along the vertical axis.

Seeking evidence for a difference between right-handers and left-handers in the processing of the terms LEFT and RIGHT, Olson and Laxar (1974) found that left-handers showed no sign of asymmetry in such processing, unlike right-handers, whose internal representation of right appeared to be less complex than that of left (Olson \& Laxar, 1973). However, the left-handed subjects in Olson and Laxar's (1974) experiment were not screened for hand dominance, but simply reported that they were sinistral. A more stringent selection of experimental subjects may bear out in left-handers a binary polar representation of the horizontal dimension that is opposite in sign to that 
of right-handers. On the contrary, ambidextrous subjects (i.e., subjects who show no hand dominance) should not exhibit any sign of asymmetry in processing the terms LEFT and RIGHT. The current research explored the contention that the term ABOVE on the vertical dimension is the referential term for right-handers, left-handers, and ambidextrous subjects, whereas the referential term on the horizontal dimension for left-handers and righthanders is that corresponding to the dominant hand (RIGHT for right-handers and LEFT for left-handers). Furthermore, according to this model, no sign of asymmetry is expected along the horizontal dimension for ambidextrous subjects.

\section{METHOD}

\section{Design}

Two similar experiments were conducted. One experiment tested terms of the vertical dimension (ABOVE and BELOW) and the other experiment tested terms of the horizontal dimension (LEFT and RIGHT). The general procedures were identical for both experiments, so the two are described together.

\section{Subjects \\ The participants in each experiment were 8 right-handers, 8 left- handers, and 8 ambidextrous subjects. Left-handers and right- handers were students of the University of Toronto, and ambidex- trous subjects were students of the University of Bologna. All sub- jects had normal or corrected-to-normal vision and were naive as to the purpose of the experiment. Each handedness group contained equal numbers of males and females. Handedness was assessed on the basis of Annett's (1970) questionnaire and on the subjects' demonstration of performance of the actions listed in the question- naire. Selected right-handers gave no "left" response and performed all the actions with the right hand, whereas selected left-handers gave no "right" response and performed all the actions with the left hand. On the contrary, ambidextrous subjects gave equal num- bers of "left" and "right" responses and performed the actions equally well with left and right hands. The reliability of the an- swers to the questionnaire was confirmed by asking the subjects to perform the actions described in it.}

\section{Materials and Procedure}

The stimuli and procedure for Experiment Above-Below were based on those of Chase and Clark (1971), whereas the stimuli and procedure for Experiment Left-Right were based on those of Olson and Laxar $(1973,1974)$. The stimuli for the experiments are shown in Figures 1 and 2 . The stimuli were generated on the screen of a computer that was positioned $50 \mathrm{~cm}$ in front of the subject's eyes. The subject viewed the stimulus binocularly and sat with the head restrained by a chinrest and with each hand holding a cylinder equipped with a pushbutton on its top. The two hands rested on a board, so that the right hand was on the right of the subject's midline and the left hand was on the left.

Each subject performed during two sessions that were run on separate days. Each session included four blocks; there were 16 practice trials and 96 experimental trials in each block. In both experiments half of the trials corresponded to displays of a true statement and the other half corresponded to displays of a false statement. Displays of a true statement in Experiment Above-Below were those in which the dot was above the word ABOVE or below the word BELOW; displays of a false statement in the same experiment were those in which the dot was below the word $A B O V E$ or above the word BELOW (top and bottom of Figure 1, respectively). In Experiment Left-Right displays of a true statement were those in which
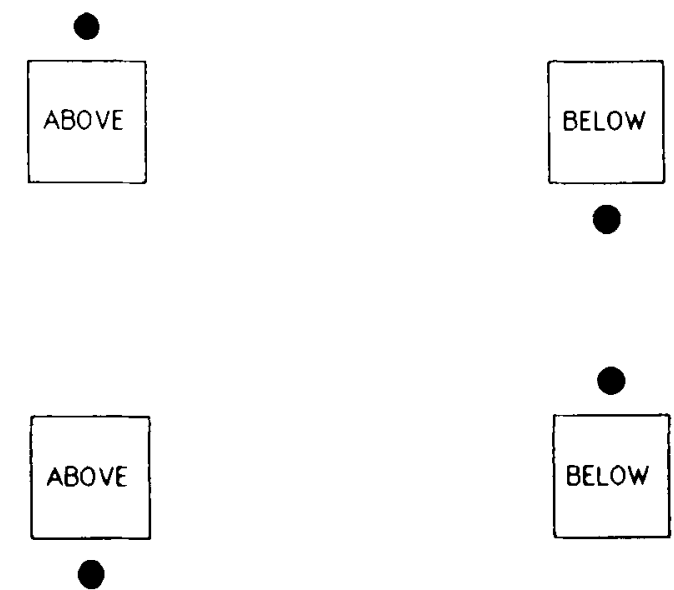

Figure 1. Displays used in Experiment Above-Below (based on displays used by Chase \& Clark, 1971).

the dot appeared to the right of the word RIGHT or to the left of the word LEFT; displays of a false statement were those in which the dot appeared to the left of the word RIGHT or to the right of the word LEFT (top and bottom of Figure 2, respectively).

Each display was preceded by a fixation target (an $\mathrm{x}$ ), which appeared at the center of the word location $1 \mathrm{sec}$ before the presentation of the stimulus display and was turned off at the display's onset. True trials and false trials of all kinds had equal probability of occurrence, so that each block included an equal number of all possible displays; otherwise, the order of presentation within each block was random. For half of the trials, the subject was asked to press the right key in response to a true statement and the left key in response to a false statement; the reverse associations were followed on the remaining trials. The order of associations between true and false statements on one side and right and left keys on the other was counterbalanced within subjects and across blocks. The subjects were asked to be both fast and accurate, but were given no feedback. Response times (RTs) were measured by a computer in a nearby room; only correct RTs longer than $100 \mathrm{msec}$ and shorter than $2.000 \mathrm{msec}$ were collected. Erroneous and off-limits RTs were noted but not replaced.

\section{RESULTS}

In both experiments mean RT was computed for each subject for each of the eight conditions resulting from the combinations of stimulus word (ABOVE or BELOW in
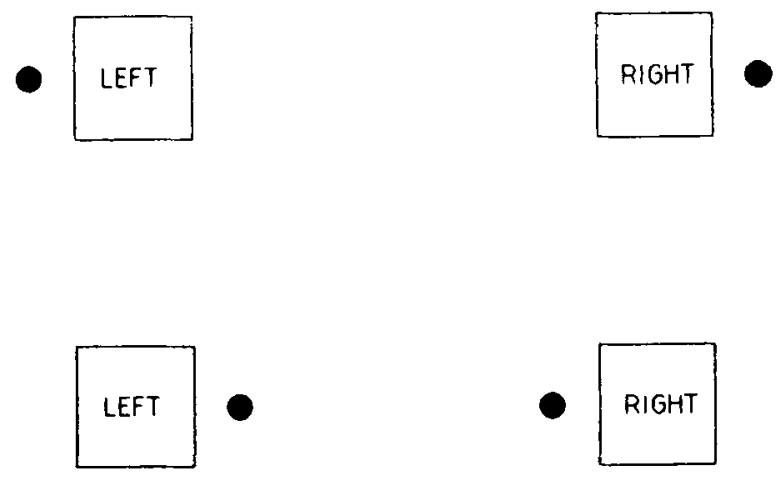

Figure 2. Displays used in Experiment Left-Right (based on displays used by Olson \& Laxar, 1973). 
Table 1

Reaction Time (in msec) as a Function of Stimulus Word (ABOVE, BELOW), Truth of Statement, and Responding Hand for Left-Handers, Right-Handers, and Ambidextrous Subjects

\begin{tabular}{|c|c|c|c|c|c|c|c|c|c|c|c|c|}
\hline \multirow[b]{3}{*}{ Hand } & \multicolumn{4}{|c|}{ Left-Handers } & \multicolumn{4}{|c|}{ Right-Handers } & \multicolumn{4}{|c|}{ Ambidextrous } \\
\hline & \multicolumn{2}{|c|}{ ABOVE } & \multicolumn{2}{|c|}{ BELOW } & \multicolumn{2}{|c|}{ ABOVE } & \multicolumn{2}{|c|}{ BELOW } & \multicolumn{2}{|c|}{ ABOVE } & \multicolumn{2}{|c|}{ BELOW } \\
\hline & True & False & True & False & True & False & True & False & True & False & True & False \\
\hline Left & 646 & 749 & 771 & 721 & 723 & 756 & 811 & 731 & 746 & 870 & 876 & 932 \\
\hline Right & 668 & 767 & 739 & 723 & 611 & 861 & 746 & 812 & 763 & 921 & 843 & 859 \\
\hline
\end{tabular}

Experiment Above-Below; LEFT or RIGHT in Experiment Left-Right), truth of statement (true or false), and responding hand (right or left). These data were submitted to two analyses of variance with repeated measures (one for each dimension examined), with handedness group (left-handers, right-handers, and ambidextrous subjects) as a between-subjects factor and the three above variables as within-subjects factors. The results are shown in Tables 1 and 2.

In Experiment Above-Below verification of statements including the word ABOVE (757 $\mathrm{msec})$ was $40 \mathrm{msec}$ faster than that of statements including the word BELOW $(797 \mathrm{msec})[F(1,21)=30.85, p<.001]$. Verification of true statements $(745 \mathrm{msec}$ ) was $63 \mathrm{msec}$ faster than that of false statements $(808 \mathrm{msec})[F(1,21)=30.66$, $p<.001]$. The interaction between these two factors was significant $[F(1,21)=49.78, p<.001]$, since the difference in favor of the statements containing the word ABOVE was significant only for true statements $[t(23)$ $=6.62, p<.001]$. Moreover, the difference between true and false responses was significant only for the statements containing the word ABOVE $[t(23)=9.48$, $p<.001]$.

There was no significant difference between left and right hands $[F(1,14)=.04]$. The interaction between type of word and responding hand was significant $[F(1,21)=$ $7.21, p<.025]$, because the left hand was faster to respond to statements containing the term ABOVE than those containing the term BELOW (748 msec and $807 \mathrm{msec}$, respectively) $[t(23)=5.03, p<.001]$, whereas for the right hand no difference was obtained between RTs to statements containing the term ABOVE and those containing the term BELOW (765 msec and $787 \mathrm{msec}$, respectively). Moreover, no significant difference was obtained between left and right hands when the subject had to respond to ABOVE or to BELOW statements. Right-handers, left-handers, and ambidextrous subjects showed the same pattern of results, with no significant difference between the three groups on any parameter.
A similar analysis of variance was carried out on the percentages of errors by using arc-sine transformations (see Table 3). The main effect of type of word was significant $[F(1,21)=23.24, p<.001]$. As for the preceding analysis of variance, the interaction between type of word and truth of statement was significant $[F(1,21)=$ $36.34, p<.001]$, since the difference in favor of the statements containing the word ABOVE compared with those containing the word BELOW ( $1 \%$ and $6 \%$, respectively) was significant for true statements $[t(23)=7.11$, $p<.001]$, but not for false statements $(3 \%$ and $2 \%$, respectively).

In Experiment Left-Right the verification of true statements was again faster than that of false statements $(803 \mathrm{msec}$ vs. $860 \mathrm{msec})[F(1,21)=31.71, p<.001]$, but the handedness group factor, the type-of-word factor, and the responding hand factor were not significant. The interaction between the two latter factors was significant $[F(1,21)=34.81, p<.001]$, because the left hand was faster in verifying or falsifying statements containing the word LEFT than those containing the word RIGHT ( $811 \mathrm{msec}$ vs. $856 \mathrm{msec}$ ) $[t(23)=3.6, p<.01]$, whereas the right hand was faster in verifying or falsifying statements containing the word RIGHT than those containing the word LEFT ( $810 \mathrm{msec}$ vs. $851 \mathrm{msec})[t(23)$ $=3.38, p<.01]$. On the other hand, the left hand was faster than the right hand at responding to the word LEFT $(811 \mathrm{msec}$ vs. $851 \mathrm{msec})[t(23)=5.03, p<.001]$, and the right hand was faster than the left hand at responding to the word RIGHT (810 msec vs. $855 \mathrm{msec})[t(23)=$ $3.81, p<.001]$. This pattern of results was exhibited by right-handers, left-handers, and ambidextrous subjects, with no significant difference between the three groups. In addition, the three groups did not perform differently as far as the responding hand was concerned, and, as a consequence, the interaction between responding hand and handedness group was not significant.

The expected interaction between handedness group and type of word was significant $[F(2,21)=11.67$, $p<.001]$. When the right-handers, left-handers, and am-

Table 2

Reaction Time (in msec) as a Function of Stimulus Word (LEFT, RIGHT), Truth of Statement, and Responding Hand for Left-Handers, Right-Handers, and Ambidextrous Subjects

\begin{tabular}{|c|c|c|c|c|c|c|c|c|c|c|c|c|}
\hline \multirow[b]{3}{*}{ Hand } & \multicolumn{4}{|c|}{ Left-Handers } & \multicolumn{4}{|c|}{ Right-Handers } & \multicolumn{4}{|c|}{ Ambidextrous } \\
\hline & \multicolumn{2}{|c|}{ LEFT } & \multicolumn{2}{|c|}{ RIGHT } & \multicolumn{2}{|c|}{ LEFT } & \multicolumn{2}{|c|}{ RIGHT } & \multicolumn{2}{|c|}{ LEFT } & \multicolumn{2}{|c|}{ RIGHT } \\
\hline & True & False & True & False & True & False & True & False & True & False & True & False \\
\hline & 629 & 82 & 771 & 868 & 8 & 841 & 854 & 8 & 754 & 981 & 8 & 100 \\
\hline Right & 820 & 760 & 848 & 749 & 805 & 920 & 664 & 862 & 939 & 863 & 903 & 846 \\
\hline
\end{tabular}


Table 3

Percentage of Correct Responses as a Function of Stimulus Word (ABOVE, BELOW), Truth of Statement, and Responding Hand for Left-Handers, Right-Handers, and Ambidextrous Subjects

\begin{tabular}{|c|c|c|c|c|c|c|c|c|c|c|c|c|}
\hline \multirow[b]{3}{*}{ Hand } & \multicolumn{4}{|c|}{ Left-Handers } & \multicolumn{4}{|c|}{ Right-Handers } & \multicolumn{4}{|c|}{ Ambidextrous } \\
\hline & \multicolumn{2}{|c|}{ ABOVE } & \multicolumn{2}{|c|}{ BELOW } & \multicolumn{2}{|c|}{ ABOVE } & \multicolumn{2}{|c|}{ BELOW } & \multicolumn{2}{|c|}{ ABOVE } & \multicolumn{2}{|c|}{ BELOW } \\
\hline & True & False & True & False & True & False & True & False & True & False & True & False \\
\hline Left & 99.8 & 99.1 & 95.9 & 98.4 & 99.0 & 97.7 & 93.0 & 99.0 & 100 & 95.6 & 90.5 & 97.2 \\
\hline Right & 99.7 & 98.4 & 90.5 & 97.9 & 99.4 & 95.0 & 89.0 & 95.2 & 97.4 & 95.2 & 98.7 & 98.8 \\
\hline
\end{tabular}

Table 4

Percentage of Correct Responses as a Function of Stimulus Word (LEFT, RIGHT), Truth of Statement, and Responding Hand for Left-Handers, Right-Handers, and Ambidextrous Subjects

\begin{tabular}{|c|c|c|c|c|c|c|c|c|c|c|c|c|}
\hline \multirow[b]{3}{*}{ Hand } & \multicolumn{4}{|c|}{ Left-Handers } & \multicolumn{4}{|c|}{ Right-Handers } & \multicolumn{4}{|c|}{ Ambidextrous } \\
\hline & \multicolumn{2}{|c|}{ LEFT } & \multicolumn{2}{|c|}{ RIGHT } & \multicolumn{2}{|c|}{ LEFT } & \multicolumn{2}{|c|}{ RIGHT } & \multicolumn{2}{|c|}{ LEFT } & \multicolumn{2}{|c|}{ RIGHT } \\
\hline & True & False & True & False & True & False & True & False & True & False & True & False \\
\hline Left & 100 & 98.0 & 90.0 & 94.0 & 96.7 & 95.8 & 89.5 & 97.0 & 94.0 & 93.0 & 93.5 & 94.5 \\
\hline Right & 95.4 & 96.5 & 99.6 & 99.6 & 89.5 & 94.0 & 99.5 & 98.5 & 97.0 & 98.0 & 97.5 & 98.5 \\
\hline
\end{tabular}

bidextrous subjects were considered as groups, it became clear that in all right-handers the verification or falsification of statements including the word RIGHT was faster than the verification or falsification of statements including the term LEFT (795 msec vs. $849 \mathrm{msec})[t(7)=2.82$, $p<.05$ ], whereas in left-handers the verification or falsification of statements containing the word LEFT was faster than the verification or falsification of statements containing the word RIGHT (759 $\mathrm{msec}$ vs. $806 \mathrm{msec}$ ) $[t(7)=3.37, p<.02]$. Seven left-handers out of 8 showed this pattern of results. In ambidextrous subjects, however, there was no significant difference between the verification or falsification of statements containing the term LEFT and those containing the term RIGHT (884 msec and $897 \mathrm{msec}$, respectively).

A similar analysis of variance was carried out on the percentages of errors by using arc-sine transformations (see Table 4). The main effect of responding hand was significant $[F(1,21)=11.52, p<.005]$, with the right hand being more accurate than the left hand $(2 \%$ and $5 \%$, respectively). Only the interaction between type of word and responding hand was significant $[F(1,21)=22.55$, $p<.001]$ : the left hand was more accurate in verifying and falsifying statements containing the term LEFT than those containing the term RIGHT (3\% and 7\%, respectively) $[t(23)=3.67, p<.01]$, and the right hand was more accurate in verifying and falsifying statements containing the term RIGHT than those containing the term LEFT $(1 \%$ vs. $3 \%)[t(23)=3.24, p<.01]$.

To verify whether subjects had more difficulty discriminating locations on the horizontal axis than discriminating locations on the vertical axis, I performed an analysis of variance with handedness group (left-handers, right-handers, and ambidextrous subjects) as a betweensubjects factor and type of dimension (horizontal and vertical) as a within-subjects factor. Only the type of dimension was significant $[F(1,21)=19.72, p<.001]$, due to the fact that in all groups verification and falsification of statements along the vertical dimension $(775 \mathrm{msec}$ ) was faster than verification and falsification of statements along the horizontal dimension $(829 \mathrm{msec})$. No other source of variability was significant. A similar analysis of variance was carried out on the percentages of errors by using arcsine transformations, but neither the main effects nor the interaction between factors were significant.

\section{DISCUSSION}

The results of the present study show that the pattern of responses of right-handers, left-handers, and ambidextrous subjects was dissimilar for judgments of horizontal dimension. Right-handers were faster in verifying and falsifying the statements containing the term RIGHT than those containing the term LEFT, whereas left-handers were faster in verifying and falsifying statements containing the term LEFT than those containing the term RIGHT. By contrast, ambidextrous subjects did not show any sign of asymmetry in the positional judgment of stimuli along the horizontal dimension. As far as the vertical dimension is concerned, right-handers, left-handers, and ambidextrous subjects were equally faster in verifying and falsifying the statements containing the term ABOVE than those containing the term BELOW. Therefore, we can conclude that although the positive polarity of the ABOVE term is independent of handedness, the positive term on the horizontal dimension in right-handers and left-handers is that corresponding to the dominant hand.

Furthermore, the results of the present study show stimulus-response (S-R) compatibility effects that are similar to those obtained when the spatial properties of the stimulus array correspond to those of the response array (Làdavas \& Moscovitch, 1984; Wallace, 1971). In all handedness groups, the left hand was faster than the right hand in verifying and falsifying the statements containing the word LEFT, whereas the right hand was faster than the left in verifying and falsifying the statements containing the word RIGHT. This type of S-R compatibility effect cannot explain the main effect found in the present 
study (i.e., the relation between the positive term on the horizontal dimension and the dominant hand), because there was no reliable interaction between handedness group and responding hand. If left-handers respond faster with their left hand and right-handers with their right hand, it should follow that left-handers respond faster to the word LEFT and right-handers respond faster to the word RIGHT. Since the two groups did not differ as far as the speed and accuracy of the two hands is concerned, the S-R compatibility effect found in the present study cannot account for the relation between the positive term on the horizontal dimension and the dominant hand.

However, this effect can be explained by assuming that the dominant or preferred hand furnishes a natural reference direction for the horizontal dimension, in the absence of any asymmetry in the physical world and in the human body. If the human organism is midsagitally symmetrical, the body is asymmetrical in terms of skill and preference. For any action one hand, one foot, one eye, and one ear performs better than the counterpart. Handedness,defined as the preference for one or the other hand or as a difference in performance between the two hands, although not the only manifestation of our asymmetrical body skill, is certainly the most striking.

Moreover, the two hands are asymmetrical not only in relation to their preferential use, but also in relation to their mental representation (Sekiyama, 1982). In righthanders the right hand's image may be generated more easily than the left hand's image, since judgments for right hands are faster than those for left hands.

Therefore, since the two hands are asymmetrical in relation to their preferential use and their mental representation, it is possible to assume that left-right hand discrimination plays an important role in learning discriminations along the horizontal dimension (Corballis $\&$ Beale, 1976). That the differentiation of the two hands along the horizontal dimension is an important factor in distinguishing left from right is supported by many studies. Benton (1959) showed that accuracy in left-right discriminations is higher when the preference for one or the other hand is more extreme. Ambidextrous subjects, who do not show any preference and any significant difference in the performance of the two hands, obtained the lowest scores in left-right discrimination tests. Furthermore, it seems that the general training in asymmetrical body skills has a specific influence in the ability to discriminate left and right (Hill, McCullum, \& Sceau, 1963). Gesell and Ames (1947) also demonstrated that the development of left-right discrimination is roughly parallel to the development of handedness. Handedness develops gradually over the first years of life until about 8 years of age, and the child learns to label the sides of his/her own body at about 6 years and keeps improving the repertoire of left-right skills over the next 4 years.

Thus, considering the preferential linkage between handedness and left-right discrimination, we can conclude that the dominant or preferred hand can furnish a reference direction for judgments related to the horizontal dimension. This can explain why right-handers are faster at verifying and falsifying statements containing the word RIGHT than those containing the word LEFT, and lefthanders are faster at verifing and falisfying statements containing the word LEFT than those containing the word RIGHT. By contrast, due to an inherent asymmetry along the vertical dimension in the physical world, there is no reason for having body coordinates as directional references for this dimension. This explains why right-handers, left-handers, and ambidextrous subjects were equally faster in verifying and falsifying statements containing the term ABOVE than those containing the term BELOW.

This study confirms also the well-documented findings that adults have more difficulty in making locational judgments within the horizontal than the vertical dimension (Farrell, 1979; Maki et al., 1979; Sholl \& Egeth, 1981). In fact, verification of statements along the vertical dimension was faster than verification of statements along the horizontal dimension. Furthermore, the results confirm those obtained by Maki et al. (1979), who found that people having less symmetrical nervous systems (i.e., righthanded subjects) and people having more symmetrical nervous systems (i.e., left-handed and ambidextrous subjects) did not perform differently in judging the horizontal dimension and the vertical dimension. Therefore, we can conclude that handedness is not responsible for the different discriminability between horizontal and vertical dimensions, but only for the different discriminability between the terms LEFT and RIGHT.

\section{REFERENCES}

ANNET, M. (1970). A classification of hand preference by association analysis. British Joumal of Psychology, 61, 303-321.

Benton, A. L. (1959). Right-left discrimination and finger vocalization: Development and pathology. New York: Hoeber-Harper.

Chase, W. G., \& Clark, H. H. (1971). Semantics in the perception of verticality. British Joumal of Psychology, 63, 311-326.

Clark, H. H. (1973). Space, time, semantics, and the child. In T. E. Moore (Ed.), Cognitive development and the acquisition of language New York: Academic Press.

Corballis, M. C., Beale, I. L. (1976). The psychology of left and right. Hillsdale, NJ: Erlbaum.

FARRELL, W. S. (1979). Coding left and right. Journal of Experimental Psychology: Human Perception \& Performance, 5, 42-51.

Gesell, A., \& AMES, L. B. (1947). The development of handedness. Journal of Genetic Psychology, 70, 155-175.

Hill, S. D., McCullum, A. H., \& Sceau, A. G. (1963). Relation of training in motor activity to development of right-left directionality in mentally retarded children: Exploratory study. Perceptual \& Motor Skills, 24, 363-366.

LÁdavas, E. \& Moscovitch, M. (1984). Must egocentric and environmental frames of reference be aligned to produce spatial $S-R$ compatibility effects? Joumal of Experimental Psychology: Human Perception \& Performance, 10, 205-215.

Maki, R. H., Grandy, C. A., \& Hauge, G. (1979). Why is telling right from left more difficult than telling above from below? Journal of Experimental Psychology: Human Perception \& Performance, 5, 52-67.

OLSON, G. M., \& LAXAR, K. (1973). Asymmetries in processing the 
terms "right" and "left." Joumal of Experimental Psychology, 100, 284-290.

Olson, G. M., \& LAXAR, K. (1974). Processing the terms "right" and "left": A note on left-handers. Journal of Experimental Psychology, 102, $1135-1137$.

SEKIYAMA, K. (1982). Kinesthetic aspects of mental representations in the identification of left and right hands. Perception \& Psychophysics, 32, 89-95.

Seymour, P. H. K. (1969). Response latencies in judgements of spatial location. British Journal of Psychology, 60, 31-39.
Seymour, P. H. K. (1974). Stroop interference with response, comparison, and encoding stages in a sentence-picture comparison task. Memory \& Cognition, 2, 19-26.

SHOLl, M. J., \& EGETh, H. E. (1981). Right-left confusion in the adult: A verbal labeling effect. Memory \& Cognition, 9, 339-350.

WALLACE, R. J. (1971). S-R compatibility and the idea of a response code. Joumal of Experimental Psychology, 93, 163-168.

(Manuscript received May 19, 1986;

revision accepted for publication October 29, 1987.) 